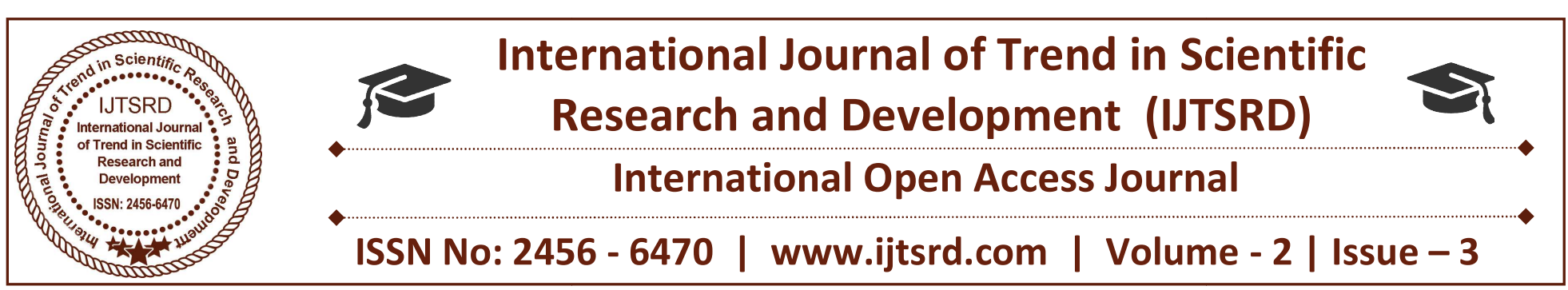

\title{
Design and Fabrication of Turning Fixture and Drilling Jig for Exhaust Manifold
}

\author{
Mr. Aditya Arvind Yadav, Mr. Akshay Vilas Arekar, \\ Mr. Sushant Subhash Chougule, Mr. Shrinath Yallapa Shirage, Mr. Yogesh Rajendra Ghodake \\ B.E., Department of Mechanical Engineering,
}

Sau. Sushila Danchnad Ghodawat Charitable Trust's, Sanjay Ghodawat Institutes,

Atigre, Kolhapur, Maharashtra, India

Dr. S. G. Bhatwadekar

Professor and Head, Department of Mechanical Engineering,

Sau. Sushila Danchnad Ghodawat Charitable Trust's, Sanjay Ghodawat Institutes,

Atigre, Kolhapur, Maharashtra, India

\section{ABSTRACT}

The successful running of any mass production depends upon the interchangeability to facilitate easy assembly and reduction of unit cost. Mass production methods demand a fast and easy method of positioning work for accurate operations on it. Jigs and fixtures are production tools used to accurately manufacture duplicate and interchangeable parts. Jigs and fixtures are specially designed so that large numbers of components can be machined or assembled identically, and to ensure interchangeability of components. To eliminate marking, punching, positioning, alignments etc. It will make easy, quick and consistently accurate locating, supporting and clamping the blank in alignment of the cutting tool. Guidance to the cutting tool like drill, reamer etc. Increase in productivity and maintain product quality consistently. It will reduce operator's labour and skill - requirement, Also reduce measurement and its cost .Enhancing technological capacity of the machine tools. Reduction of overall machining cost and also increases in interchangeability.

Keywords: turning fixture, drilling jig, design

\section{INTRODUCTION}

The successful running of any mass production depends upon the interchangeability to facilitate easy assembly and reduction of unit cost. Mass production methods demand a fast and easy method of positioning work for accurate operations on it. Jigs and fixtures are production tools used to accurately manufacture duplicate and interchangeable parts. Jigs and fixtures are specially designed so that large numbers of components can be machined or assembled identically, and to ensure interchangeability of components. The details of jigs and fixtures is as given below:

JIG:

It is a work holding device that holds, supports and locates the work piece and guides the cutting tool for a specific operation. Jigs are provided with tool guiding elements such as drill bush. These direct the tool to the correct position on the work piece. A jig is a type of tool used to control the location and motion of another tool. A jig's primary purpose is to provide repeatability, accuracy, and Interchangeability in the manufacturing of products. A device that does both functions (holding the work and guiding a tool) is called a jig. 


\section{FIXTURE:}

Fixture holds the work piece securely in the correct position with respect to the machine during operation. There is sometimes a provision with respect to the work piece, but the tool is not guided as in a jig. Fixtures are often clamped to the machine table. It is a work holding device that holds supports and locates the work piece for a specific operation but does not guide the cutting tool. It provides only a reference surface or a device. The main purpose of a fixture is to locate and in some cases hold a work piece during either a machining operation or some other industrial process. A jig differs from a Fixture in that it guides the tool to its correct position in addition to locating and supporting the work piece. The elements of the jigs and fixtures are as follows:

\section{Locating elements}

These position the work piece accurately with respect to the tool guiding or setting elements in the fixture.

\section{Clamping elements}

These hold the work piece securely in the located position during operation.

\section{Tool guiding and setting elements}

These aid guiding or setting of the tools in correct position with respect to the work piece. Drill bushings guide the drills accurately to the work piece. Milling fixtures use setting pieces for correct positioning of milling cutters with respect to the work piece

\section{Advantages of jig and fixture}

Productivity - Jig and fixtures eliminate individual marking, positioning and frequent checking. This reduces operation time and increase the productivity.

Interchangeability - Jig and fixtures facilitate uniform quality in manufacture. There is no need for selective assembly. Any part of the machine fit properly in to the assembly. And all similar components are interchangeable.

Skill reduction - Jig and fixtures simplify locating and clamping of the work piece. Tool guiding elements ensure clamping of the work piece. There is no need for skillful setting of the work piece of tool. Any average person can be trained to use Jig and fixtures. It saves the labour cost.

Cost reduction - higher production, reduction in scrap, easy assembly and savings in labour costs result in substantial reduction in the cost of work pieces produced with jigs and fixtures.

\section{OBJECTIVE:}

The basic purposes of developing and using drilling jigs and turning fixtures for batch production in machine shops are:

1. To eliminate marking, punching, positioning, alignments etc.

2. Easy, quick and consistently accurate locating, supporting and clamping the blank in alignment of the cutting tool

3. Guidance to the cutting tool like drill, reamer etc.

4. Increase in productivity and maintain product quality consistently

5. To reduce operator's labour and skill requirement

6. To reduce measurement and its cost

7. Enhancing technological capacity of the machine tools

8. Reduction of overall machining cost and also increases in interchangeability.

Factors to be considered for design of Jigs and Fixtures

1. Component- Design to be studied carefully Ensure work is performed in a proper sequence Maximum operations should be performed on a machine in single setting.

2. Capacity of the machine-Careful consideration to be performed on type and capacity of machine.

3. Production requirements-Design to be made on basis of actual production requirements. Then comes decision on manual and automatic tooling arrangements.

\section{MATERIALS USED FOR MANUFACTURING OF JIGS AND FIXTURE:}

Jigs and fixture are made from a variety of materials, some of which can be hardened to resist wear. It is sometimes necessary to use nonferrous metals like phosphor bronze to reduce wear of the mating parts, 
or nylons or fiber to prevent damage to the work piece.

\section{High Speed Steel(HSS):}

These contain $18 \%$ (or $22 \%$ ) tungsten for toughness and cutting strength, $4.3 \%$ chromium for better hardenability and wear resistance and $1 \%$ vanadium for retention of hardness at high temperature (red hardness ) and impact resistance. HSS can be air or oil hardened to RC64-65 and are suitable for cutting tools such as drills, reamers an63.

\section{Die steels :}

These are also called high carbon (1.5-2.3\%) high chromium $(12 \%)(\mathrm{HCHC})$ cold working steels and are used cutting press tools and thread forming rolls. Hot die steel with lesser carbon $(.35 \%)$ and chromium $(5 \%)$ but alloyed with molybdenum (1\%) and vanadium $(.3-1 \%)$ for retention of hardness at high temperature are used for high temperature are used for high temperature work like forging, casting and extrusion.

\section{Carbon Steel:}

These contain $0.85-1.18 \%$ carbon and can be oil hardened to RC62-63.The parts of jigs and fixtures like bushing and locaters, which are subjected to heavy wear can also be made from carbon steels and hardened.

\section{Collet steels (spring steels):}

These contain about $1 \%$ carbon and $0.5 \%$ manganese. Spring steels are usually tempered to RC 47 hardness.

\section{Oil Hardening Non-shrinking tool steel(OHNS)}

These contain $0.9-1.1 \%$ carbon, $0.5-2 \%$ tungsten and $0.45-1 \%$ carbon. These are used for fine parts such as taps, hand reamers, milling cutters, engraving tools, and intricate press tools which cannot be ground after hardening ( $\mathrm{RC} 62)$

\section{Case Hardening Steels:}

These can be carburized and case hardened to provide 0.6-1.5\%thick, hard (RC 59-63) exterior.17Mn1Cr95 steel with $1 \%$ manganese and $0.95 \%$ chromium is widely used.15Ni2Cr1Mo15 steel with additional nickel (2\%) reduces thermal expansion up to 373K.Case hardening steels are suitable for parts which require only local hardness on small wearing surfaces where costlier, difficult to machine full hardening tool steels are not warranted.

\section{High Tensile Steel}

These can be classified into medium carbon steels with $0.45 \%-0.65 \%$ carbon(En24) and alloy steels like 40Ni2Cr1Mo28(En24).the tensile strength can be increased up to $125 \mathrm{~kg} / \mathrm{mm} 2$ ( $\mathrm{RC} \mathrm{40)}$ by tempering. Medium carbon steels are used widely for fasteners and structural work while alloy steels are used for high stress applications like press rams.

\section{Mild Steel:}

It is the cheapest and most widely used material in jigs and fixture. It contains less than $0.3 \%$ carbon. It is economical to make parts which are not subjected too much wear and are not highly stressed from mild steel.

\section{Cast Iron:}

It contains $2-2.5 \%$ carbon. As it can withstand vibrations well, it is used widely in milling fixtures. Self-lubricating properties make cast iron suitable for machine slides and guide-ways. The ingenious shaping for a casting and pattern can save a lot of machining time. Although, the strength of cast iron is only half the strength of mild steel, a wide variety of grades have been developed. Nodular cast iron is as strong as mild steel, while mechanize casting have heat resistant, wear resistance, and corrosion resistant grades.

\section{Steel castings:}

These combine the strength of steel and capability of casting.

\section{Nylon and Fiber}

These are usually used as soft as soft lining for clamps to prevent denting or damage to the work piece under high clamping force. Nylon or fiber pads are screwed or stuck to mild steel clamps.

\section{Phosphor Bronze}

It is widely used for replaceable nuts in screw operated feeding and clamping systems. Generally screw operated feeding and clamping systems. Generally screw making is time consuming and costly .So, their wear is minimized by using softer, shorter phosphor

Bronze mating nuts. These can be replaced periodically. Phosphor bronze is also used in application calling for corrosion resistance, like boiler valves. 


\section{METHODOLOGY:}

1. Design of component studied carefully.

2. Maximum operations should be performed on a machine in single setting.

3. Careful consideration to be performed on type and capacity of machine.

4. Design to be made on basis of actual production requirement.

5. Location should ensure equal distribution of forces throughout all sequence of operation.

6. To study about easy and quick loading of work piece.

7. To study about clamping arrangements. The clamping should not cause any deformation to the work piece.

8. To study about features of clamps.

a. Clamping pressure should be low.

b. Movement of clamp should be minimum.

c. Sufficiently robust to avoid bending.

9. To study about clearance between jig and component.

10. To study about tool guiding and cutter setting.

a. By adjusting the machine or using cutter setting block, the cutter is set relative to the work in fixture.

b. The drill bushes fitted on jig plates guides the tools.

11. To study about rigidity and vibration.

a. Must possess enough rigidity robustness.

b. Should not vibrate as it may lead to unwanted movement of work piece and tools.
Component

PIPE EGR COOLER INLET

MAHINDRA AND MAHINDRA LTD.

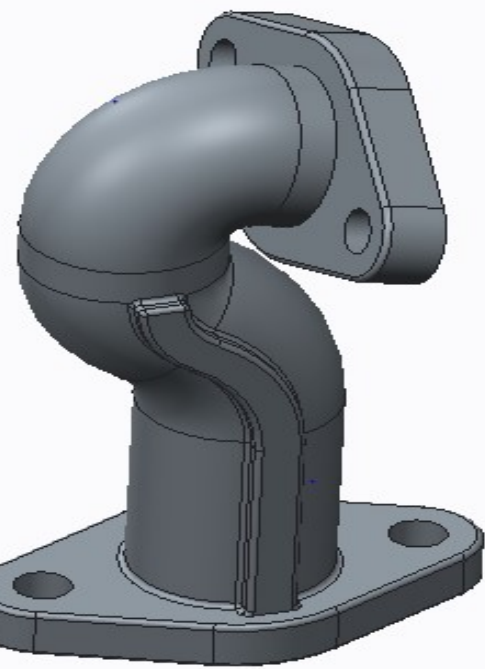

MATERIAL: - GREY CAST IRON FG 250

Operation perform on the component

1. Facing exhaust manifold side

2. Drilling exhaust manifold side

3. Angular face milling cooler side

4. Drilling cooler side

5. Spot facing one side hole

6. Spot facing milling another side hole

7. Spot facing of angular side cooler side

\section{MACHINE SPECIFICATIONS:}

1. Lathe machine

2. Drilling machine

3. Milling machine

1. Milling Machine

Manufacture's Name: HMT Ltd, Hyderabad.

Type: BTM- 136

Table size- 1500 x $500 \mathrm{~mm}$

No. of speeds- 16

Max. Load- 600 kg

$\mathrm{L} \times \mathrm{W} \times \mathrm{H}=6000 \times 4500 \times 3000$

Self-wt. - $9000 \mathrm{~kg}$ 


\section{Drilling Machine}

Manufacture's Name: HMT, Bangalore.

Type: RM- 65

Drill capacity- $60 \mathrm{~mm}$ dia. in C.I.

No. of spindle speeds- 12 nos

Range- 40 to 1800

No. of feeds- 6

$\mathrm{L} \times \mathrm{H} \times \mathrm{H}=3000 \times 1280 \times 3550$

Weight- $3700 \mathrm{kgs}$

\section{ELEMENTS FOR JIG AND FIXTURE:}

\section{SCREW CLAMPS:}

Screw clamps are threaded devices with knurled collar, hand knob, Tommy bar or spanner flats for rotating and tightening the screw. The clamping area of a screw clamp can be increased by a provision for pad. The clamping pad is free to rotate on the pivot. This eliminates friction between the work piece and the pad. The clamping pad remain stationary on the work piece while the screw rotates and rubs on the conical seat of the pad.

2. C WASHER:

The $C$ washer is a strap clamp with an open slot and is simple and quick in operation

\section{PLAIN BUSH:}

It is used when a hole is to be produced only by one tool.

\section{V LOCATER:}

$\mathrm{V}$ Locater is used to locate from cylindrical or part cylindrical features on a work piece. These may be fixed or sliding. An adjustable v- locator should be guided by guide plate to constrain its movement along the axis of $\mathrm{v}$. the guides must be dowelled to prevent shifting during operation. The side of the $\mathrm{v}$ face is sometimes inclined slightly to provide downward.

\section{DESIGN OF JIGS AND FIXTURE}

Turning fixture

1. Facing exhaust manifold side

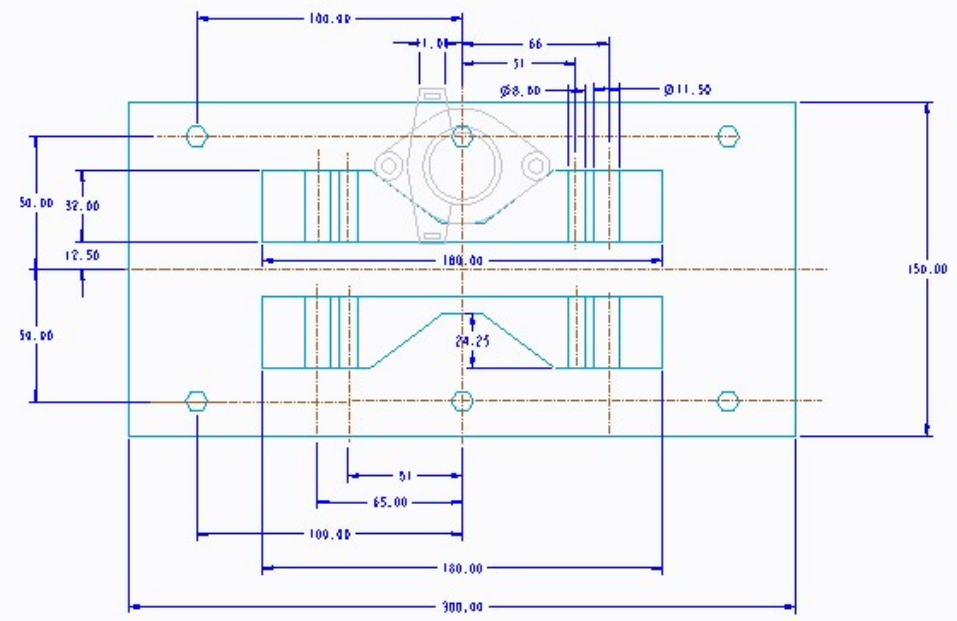

2. Drill jig

Drilling exhaust manifold side Drill dia $8.5 \mathrm{~mm}$

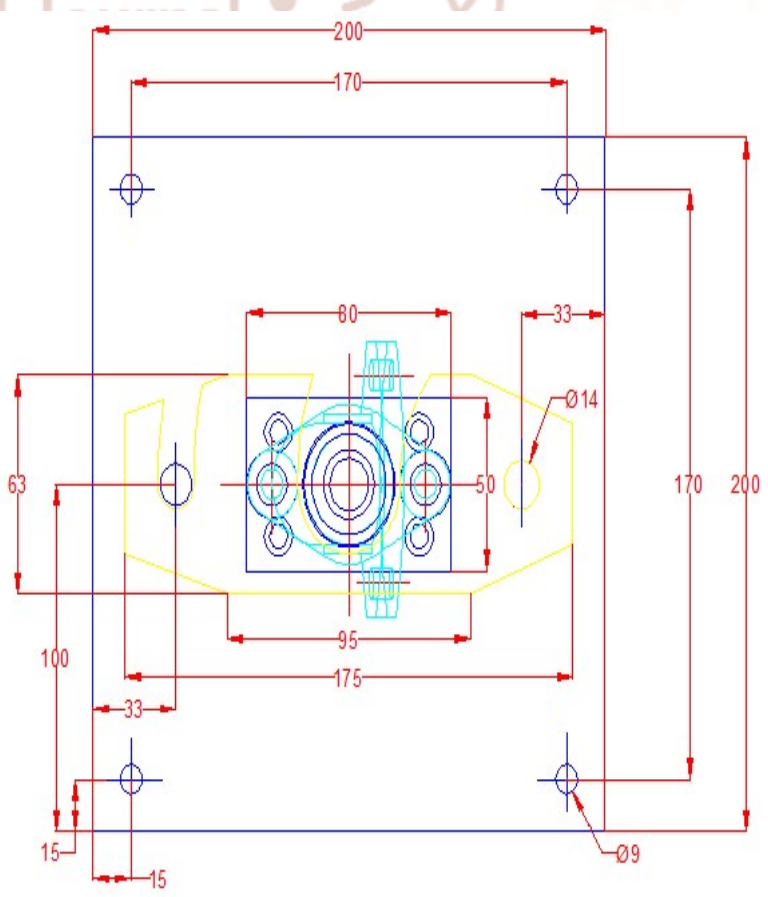


International Journal of Trend in Scientific Research and Development (IJTSRD) ISSN: 2456-6470 CATIA DESIGN OF TURNING FIXTURE AND DRILLING JIG

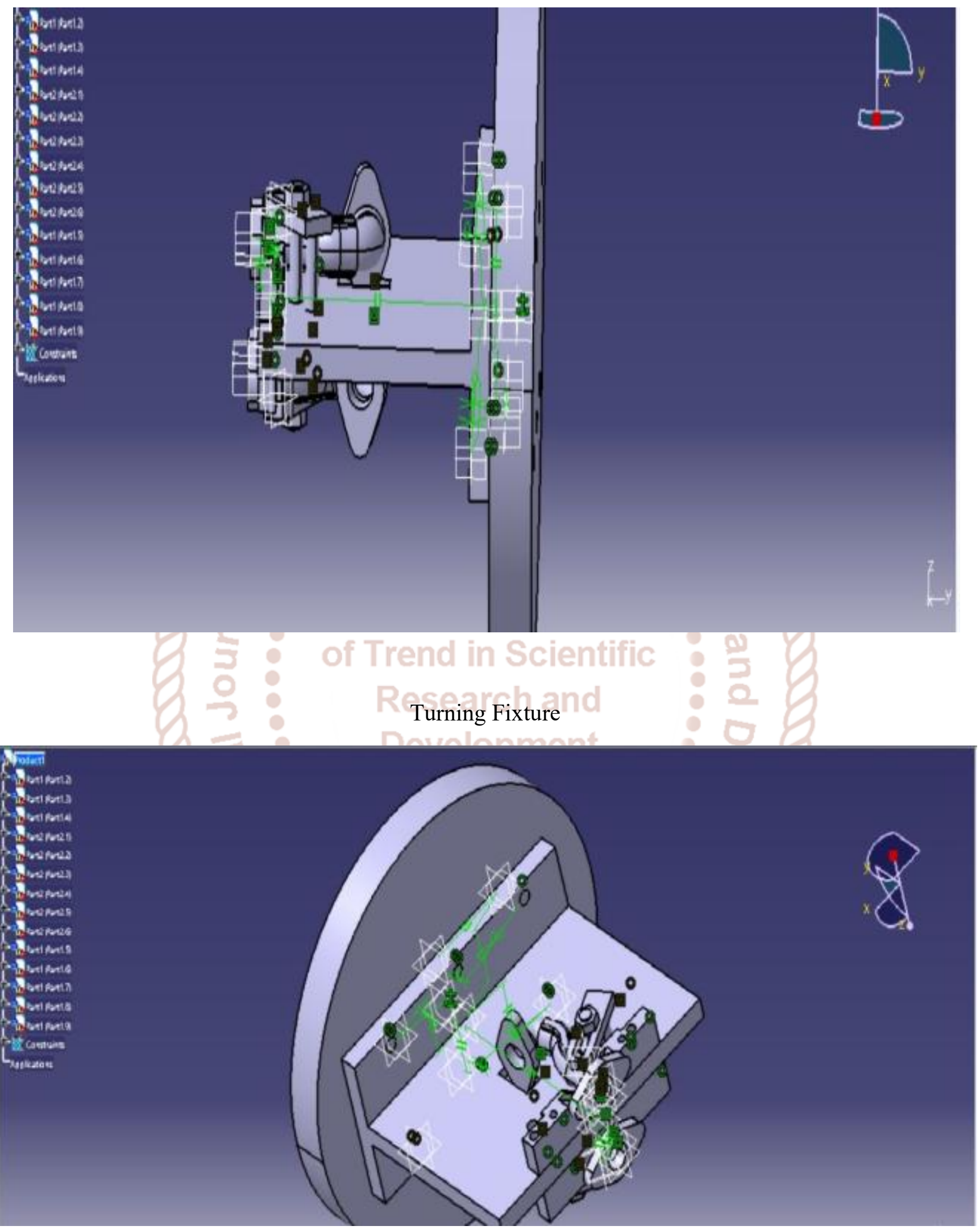

Turning fixture 


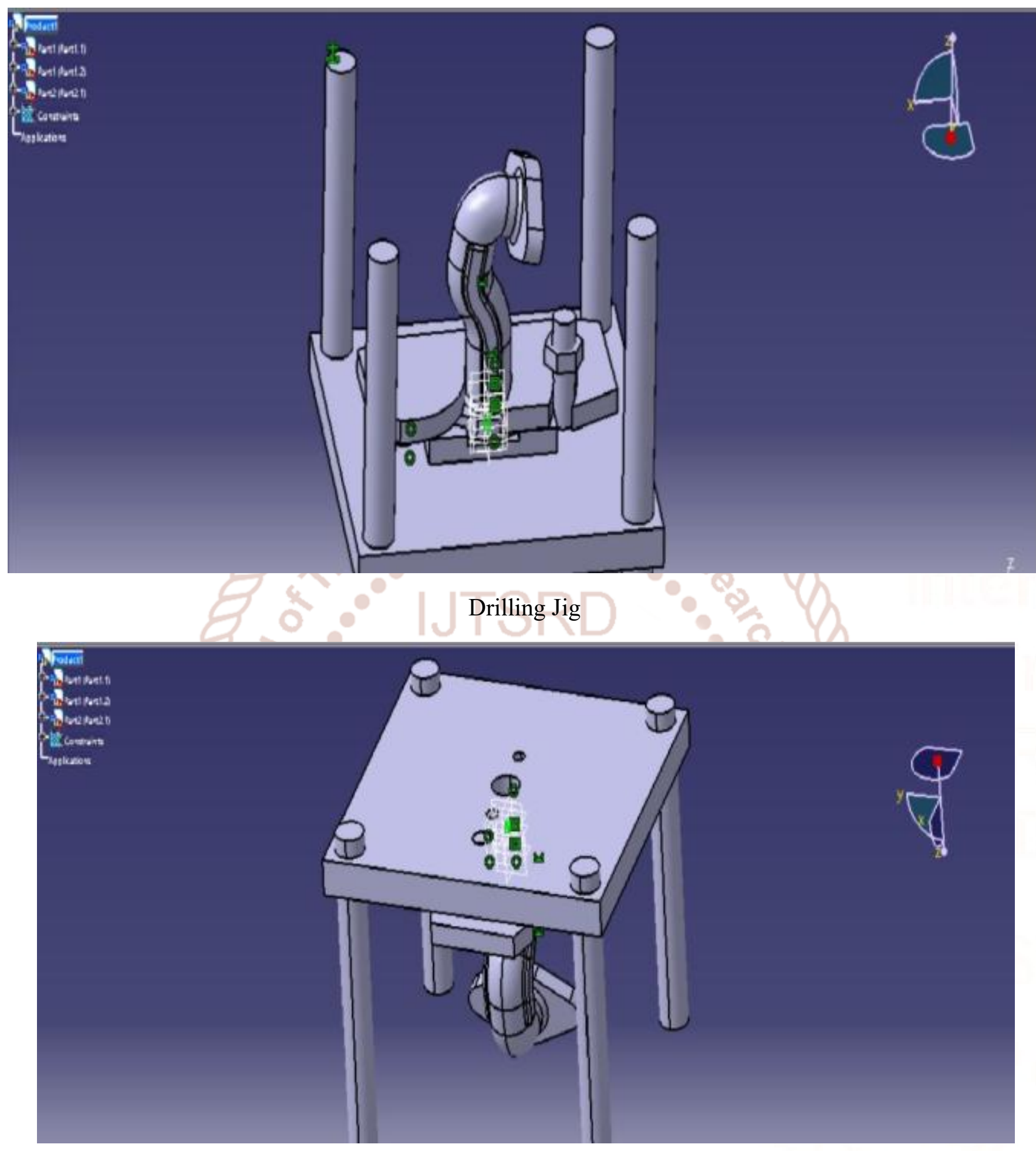

Drilling Jig

\section{CONCLUSION}

To eliminate marking, punching, positioning, alignments etc. It will make easy, quick and consistently accurate locating, supporting and clamping the blank in alignment of the cutting tool. Guidance to the cutting tool like drill, reamer etc. Increase in productivity and maintain product quality consistently. It will reduce operator's labour and skill
- requirement, Also reduce measurement and its cost .Enhancing technological capacity of the machine tools. Reduction of overall machining cost and also increases in interchangeability. 


\section{REFERENCES:}

1) Jig and fixture design manual by P.H. JOSHI.

2) An Introduction To Jig and Tool design by Kempster, M.H.A published by ELBS

3) Hand Book Of Jigs And Fixture design by Boyes, William E published by SME

4) Jigs And Fixture Design Manual by Henrickson, E.K published by INDUSTRIAL PRESS

5) Jigs And Fixture Hand Book by Goroshkin, A.K. published by MIR

6) Introduction To Jig And Tool Design by Kempster, M.H.A published by VIVA 